\title{
A REFINEMENT OF A DOUBLE INEQUALITY FOR THE GAMMA FUNCTION
}

\author{
FENG QI AND BAI-NI GUO
}

\begin{abstract}
In the paper, we present a monotonicity result of a function involving the gamma function and the logarithmic function, refine a double inequality for the gamma function, and improve some known results for bounding the gamma function.
\end{abstract}

\section{INTRODUCTION}

In [10], the following double inequality was complicatedly procured: For $x \in$ $(0,1)$

$$
\frac{x^{2}+1}{x+1}<\Gamma(x+1)<\frac{x^{2}+2}{x+2}
$$

where $\Gamma(x)$ stands for the classical Euler's gamma function which may be defined for $x>0$ by

$$
\Gamma(x)=\int_{0}^{\infty} t^{x-1} e^{-t} \mathrm{~d} t
$$

The aim of this paper is to simply and concisely generalize, refine and sharpen the double inequality (1.1).

Our main results may be stated as the following theorem.

Theorem 1. The function

$$
\frac{\ln \Gamma(x+1)}{\ln \left(x^{2}+1\right)-\ln (x+1)}
$$

is strictly increasing on $(0,1)$, with the limits

$$
\lim _{x \rightarrow 0^{+}} \frac{\ln \Gamma(x+1)}{\ln \left(x^{2}+1\right)-\ln (x+1)}=\gamma
$$

and

$$
\lim _{x \rightarrow 1^{-}} \frac{\ln \Gamma(x+1)}{\ln \left(x^{2}+1\right)-\ln (x+1)}=2(1-\gamma) .
$$

As a result, the double inequality

$$
\left(\frac{x^{2}+1}{x+1}\right)^{\alpha}<\Gamma(x+1)<\left(\frac{x^{2}+1}{x+1}\right)^{\beta}
$$

2010 Mathematics Subject Classification. Primary 26A48, 33B15; Secondary 26D15.

Key words and phrases. monotonicity, generalization, refinement, sharpening, inequality, gamma function, Descartes' Sign Rule, open problem, conjecture.

The first author was supported in part by the Science Foundation of Tianjin Polytechnic University.

This paper was typeset using $\mathcal{A M}_{\mathcal{M}}$-LATEX. 
holds on $(0,1)$ if and only if $\alpha \geq 2(1-\gamma)$ and $\beta \leq \gamma$, where $\gamma=0.57 \cdots$ stands for Euler-Mascheroni's constant. Consequently, the double inequality

$$
\begin{aligned}
{\left[\frac{(x-\lfloor x\rfloor)^{2}+1}{x-\lfloor x\rfloor+1}\right]^{\alpha} \prod_{i=0}^{\lfloor x\rfloor-1}(x-i)<\Gamma(x+1) } & \\
& <\left[\frac{(x-\lfloor x\rfloor)^{2}+1}{x-\lfloor x\rfloor+1}\right]^{\beta} \prod_{i=0}^{\lfloor x\rfloor-1}(x-i)
\end{aligned}
$$

holds for $x \in(0, \infty) \backslash \mathbb{N}$ if and only if $\alpha \geq 2(1-\gamma)$ and $\beta \leq \gamma$, where $\lfloor x\rfloor$ represents the largest integer less than or equal to $x$.

In Section 2, we cite three lemmas for proving in Section 3 Theorem 1. In Section 4, we compare Theorem 1 with several known results and pose some open problems and conjectures.

\section{LEMMAS}

In order to prove Theorem 1, we need the following lemma which can be found in [3], [18, pp. 9-10, Lemma 2.9], [19, p. 71, Lemma 1] or closely-related references therein.

Lemma 1. Let $f$ and $g$ be continuous on $[a, b]$ and differentiable on $(a, b)$ such that $g^{\prime}(x) \neq 0$ on $(a, b)$. If $\frac{f^{\prime}(x)}{g^{\prime}(x)}$ is increasing (or decreasing) on $(a, b)$, then so are the functions $\frac{f(x)-f(b)}{g(x)-g(b)}$ and $\frac{f(x)-f(a)}{g(x)-g(a)}$ on $(a, b)$.

We also need the following elementary conclusions.

Lemma 2. For $x \in(0,1)$, we have

$$
\begin{aligned}
x^{4}+4 x^{3}-2 x^{2}-4 x-3 & <0, \\
(x-1)\left(x^{2}+2 x-1\right)-(x+1)\left(x^{2}+1\right) \ln \frac{x^{2}+1}{x+1} & >0, \\
x^{6}+6 x^{5}-3 x^{4}-16 x^{3}-21 x^{2}-6 x-1 & <0, \\
x^{5}+5 x^{4}-2 x^{3}-8 x^{2}-7 x-1 & <0, \\
5 x^{7}+34 x^{6}+27 x^{5}-62 x^{4}-205 x^{3}-198 x^{2}-83 x-6 & <0 .
\end{aligned}
$$

Proof. For our own convenience, denote the functions above by $h_{i}(x)$ for $1 \leq i \leq 5$ on $[0,1]$ in order.

By Descartes' Sign Rule, the function $h_{1}(x)$ has just one possible positive root. Since $h_{1}(1)=-4$ and $h_{1}(2)=29$, the function $h_{1}(x)$ is negative on $[0,1]$.

A straightforward calculation gives

$$
\frac{\mathrm{d}}{\mathrm{d} x}\left[\frac{h_{2}(x)}{(x+1)\left(x^{2}+1\right)}\right]=\frac{(x-1) h_{1}(x)}{(x+1)^{2}\left(x^{2}+1\right)^{2}},
$$

so the function $\frac{h_{2}(x)}{(x+1)\left(x^{2}+1\right)}$ is strictly increasing on $[0,1]$. Due to $h_{2}(0)=1$, it is derived that $h_{2}(x)>0$ on $(0,1)$.

Since

$$
\begin{array}{lll}
h_{3}(1)=-40, & h_{3}(3)=1304, & h_{4}(1)=-12, \\
h_{4}(2)=49, & h_{5}(1)=-488, & h_{5}(2)=84,
\end{array}
$$


using Descartes' Sign Rule again yields the negativity of the functions $h_{i}(x)$ for $3 \leq i \leq 5$ on $(0,1)$. The proof of Lemma 2 is complete.

For our own convenience, we also recite the following double inequality for polygamma functions $\psi^{(k)}(x)$ on $(0, \infty)$.

Lemma 3. The double inequality

$$
\frac{(k-1) !}{x^{k}}+\frac{k !}{2 x^{k+1}}<(-1)^{k+1} \psi^{(k)}(x)<\frac{(k-1) !}{x^{k}}+\frac{k !}{x^{k+1}}
$$

holds for $x>0$ and $k \in \mathbb{N}$.

For the proof of the inequality (2.1), please refer to [5, p. 131], [6, p. 223, Lemma 2.3], [7, p. 107, Lemma 3], [8, p. 853], [12, p. 55, Theorem 5.11], [13, p. 1625], [16, p. 79], [17, p. 2155, Lemma 3] and closely-related references therein.

\section{Proof of Theorem 1}

Now we are in a position to prove our main results in Theorem 1.

It is easy to see that

$$
\begin{gathered}
\frac{\ln \Gamma(x+1)}{\ln \left(x^{2}+1\right)-\ln (x+1)}=\frac{\frac{1}{x-1} \ln \Gamma(x+1)}{\frac{1}{x-1} \ln \frac{x^{2}+1}{x+1}}=\frac{\ln \sqrt[x-1]{\Gamma(x+1)}}{\ln \sqrt[x-1]{\frac{x^{2}+1}{x+1}}} \\
=\frac{\ln \sqrt[x-1]{\Gamma(x+1)}-\ln \sqrt[0-1]{\Gamma(0+1)}}{\ln \sqrt[x-1]{\frac{x^{2}+1}{x+1}}-\ln \sqrt[0-1]{\frac{0^{2}+1}{0+1}}}=\frac{f(x)-f(0)}{g(x)-g(0)}
\end{gathered}
$$

where

$$
f(x)=\ln \sqrt[x-1]{\Gamma(x+1)} \quad \text { and } \quad g(x)=\ln \sqrt[x-1]{\frac{x^{2}+1}{x+1}}
$$

on $[0,1]$. Easy computation and simplification yield

$$
\frac{f^{\prime}(x)}{g^{\prime}(x)}=\frac{(x+1)\left(x^{2}+1\right)[(x-1) \psi(x+1)-\ln \Gamma(x+1)]}{(x-1)\left(x^{2}+2 x-1\right)-(x+1)\left(x^{2}+1\right) \ln \frac{x^{2}+1}{x+1}}
$$

and

$$
\frac{\mathrm{d}}{\mathrm{d} x}\left[\frac{f^{\prime}(x)}{g^{\prime}(x)}\right]=\frac{(1-x)\left(x^{4}+4 x^{3}-2 x^{2}-4 x-3\right) q(x)}{\left[(x-1)\left(x^{2}+2 x-1\right)-(x+1)\left(x^{2}+1\right) \ln \frac{x^{2}+1}{x+1}\right]^{2}},
$$

where

$$
\begin{aligned}
q(x)=\ln \Gamma(x+1) & -(x-1) \psi(x+1)-\frac{(x+1)\left(x^{2}+1\right)}{x^{4}+4 x^{3}-2 x^{2}-4 x-3} \\
\times & {\left[(x-1)\left(x^{2}+2 x-1\right)-(x+1)\left(x^{2}+1\right) \ln \frac{x^{2}+1}{x+1}\right] \psi^{\prime}(x+1) . }
\end{aligned}
$$

Further computation and simplification give

$$
q^{\prime}(x)=\frac{(1-x)\left(x^{2}+2 x-1\right)+(x+1)\left(x^{2}+1\right) \ln \frac{x^{2}+1}{x+1}}{\left(x^{4}+4 x^{3}-2 x^{2}-4 x-3\right)^{2}} q_{1}(x),
$$

where

$$
\begin{aligned}
& q_{1}(x)=2\left(x^{6}+6 x^{5}-3\right.\left.x^{4}-16 x^{3}-21 x^{2}-6 x-1\right) \psi^{\prime}(x+1) \\
&+(x+1)\left(x^{2}+1\right)\left(x^{4}+4 x^{3}-2 x^{2}-4 x-3\right) \psi^{\prime \prime}(x+1)
\end{aligned}
$$


and satisfies

$$
\begin{aligned}
& q_{1}^{\prime}(x)=12\left(x^{5}+5 x^{4}-2 x^{3}-8 x^{2}-7 x-1\right) \psi^{\prime}(x+1) \\
& +\left(x^{4}+4 x^{3}-2 x^{2}-4 x-3\right)\left[3\left(3 x^{2}+2 x+1\right) \psi^{\prime \prime}(x+1)\right. \\
& \left.+(x+1)\left(x^{2}+1\right) \psi^{\prime \prime \prime}(x+1)\right] .
\end{aligned}
$$

By virtue of Lemmas 2 and 3, we obtain

$$
\begin{aligned}
q_{1}^{\prime}(x)< & \left(x^{4}+4 x^{3}-2 x^{2}-4 x-3\right)\left\{(x+1)\left(x^{2}+1\right)\left[\frac{2}{(x+1)^{3}}+\frac{3}{(x+1)^{4}}\right]\right. \\
& \left.-3\left(3 x^{2}+2 x+1\right)\left[\frac{1}{(x+1)^{2}}+\frac{2}{(x+1)^{3}}\right]\right\} \\
& +12\left(x^{5}+5 x^{4}-2 x^{3}-8 x^{2}-7 x-1\right)\left[\frac{1}{x+1}+\frac{1}{2(x+1)^{2}}\right] \\
= & \frac{5 x^{7}+34 x^{6}+27 x^{5}-62 x^{4}-205 x^{3}-198 x^{2}-83 x-6}{(x+1)^{3}} \\
< & 0
\end{aligned}
$$

on $[0,1]$. So the function $q_{1}(x)$ is strictly decreasing on $[0,1]$. Since

$$
q_{1}(0)=-2 \psi^{\prime}(1)-3 \psi^{\prime \prime}(1)=3.922 \cdots
$$

and

$$
q_{1}(1)=80\left(1-\frac{\pi^{2}}{6}\right)-16 \psi^{\prime \prime}(2)=-45.128 \cdots,
$$

the function $q_{1}(x)$ has a unique zero on $(0,1)$, and so is the function $q^{\prime}(x)$. As a result, the function $q(x)$ has a unique minimum on $(0,1)$. Because of

$$
q(0)=\frac{1}{3}\left(\frac{\pi^{2}}{6}-3 \gamma\right)=-0.028 \cdots
$$

and $q(1)=0$, we obtain that $q(x)<0$ on $(0,1)$. Combining this with Lemma 2 leads to

$$
\frac{\mathrm{d}}{\mathrm{d} x}\left[\frac{f^{\prime}(x)}{g^{\prime}(x)}\right]>0
$$

on $(0,1)$, which means that the function $\frac{f^{\prime}(x)}{g^{\prime}(x)}$ is strictly increasing on $(0,1)$. Furthermore, from Lemma 1 and the equation (3.1), it follows that the function (1.3) is strictly increasing on $(0,1)$.

By L'Hospital's rule, we have

$$
\begin{aligned}
\lim _{x \rightarrow 0^{+}} \frac{\ln \Gamma(x+1)}{\ln \left(x^{2}+1\right)-\ln (x+1)} & =\lim _{x \rightarrow 0^{+}} \frac{(x+1)\left(x^{2}+1\right) \psi(x+1)}{x^{2}+2 x-1} \\
& =-\psi(1) \\
& =\gamma
\end{aligned}
$$

and

$$
\begin{aligned}
\lim _{x \rightarrow 1^{-}} \frac{\ln \Gamma(x+1)}{\ln \left(x^{2}+1\right)-\ln (x+1)} & =\lim _{x \rightarrow 1^{-}} \frac{(x+1)\left(x^{2}+1\right) \psi(x+1)}{x^{2}+2 x-1} \\
& =2 \psi(2) \\
& =2(1-\gamma) .
\end{aligned}
$$


Hence, the double inequality (1.6) and its sharpness follow.

The double inequality (1.7) may be deduced from (1.6) and the recurrent formula $\Gamma(x+1)=x \Gamma(x)$ for $x>0$. The proof of Theorem 1 is complete.

\section{REMARKS}

In this section, we compare Theorem 1 with some known results and pose several open problems and conjectures.

Remark 1. It is clear that the double inequality (1.6) refines the double inequality (1.1). Moreover, the inequality (1.6) may be rearranged as

$$
\frac{1}{x}\left(\frac{x^{2}+1}{x+1}\right)^{2(1-\gamma)}<\Gamma(x)<\frac{1}{x}\left(\frac{x^{2}+1}{x+1}\right)^{\gamma}, \quad x \in(0,1) .
$$

Remark 2. In [1, p. 145, Theorem 2], it was obtained that if $x \in(0,1)$, then

$$
x^{\alpha(x-1)-\gamma}<\Gamma(x)<x^{\beta(x-1)-\gamma}
$$

with the best possible constants

$$
\alpha=1-\gamma=0.42278 \cdots \text { and } \beta=\frac{1}{2}\left(\frac{\pi^{2}}{6}-\gamma\right)=0.53385 \cdots,
$$

and if $x \in(1, \infty)$, then (4.2) holds with the best possible constants

$$
\alpha=\frac{1}{2}\left(\frac{\pi^{2}}{6}-\gamma\right) \text { and } \beta=1 .
$$

In $[2$, p. 780 , Corollary], the following conclusion was established: Let $\alpha$ and $\beta$ be nonnegative real numbers. For $x>0$, we have

$$
\sqrt{2 \pi} x^{x} \exp \left[-x-\frac{1}{2} \psi(x+\alpha)\right]<\Gamma(x)<\sqrt{2 \pi} x^{x} \exp \left[-x-\frac{1}{2} \psi(x+\beta)\right]
$$

with the best possible constants $\alpha=\frac{1}{3}$ and $\beta=0$.

In [9, p. 3, Theorem 5], among other things, it was demonstrated that for $x \in$ $(0,1]$ we have

$$
\frac{x^{x[1-\ln x+\psi(x)]}}{e^{x}}<\Gamma(x) \leq \frac{x^{x[1-\ln x+\psi(x)]}}{e^{x-1}} .
$$

By the well-known software Mathematica Version 7.0.0, we can show that

(1) the double inequalities (4.1) and (4.2) are not included each other on $(0,1)$,

(2) when $x>0$ is smaller, the double inequalities (4.1) is better than (4.2),

(3) the double inequality (4.1) improves $(4.5)$ on $(0,1)$,

(4) the left-hand side inequality in (4.1) refines the corresponding one in (4.6),

(5) the right-hand side inequalities in (4.1) and (4.6) are not contained each other,

(6) when $x>0$ is smaller, the right-hand side inequality in (4.1) is better than the corresponding one in (4.6).

Remark 3. In [4, Corollary 1.2, Theorem 1.4 and Theorem 1.5], the following sharp inequalities for bounding the gamma function were obtained: For $x>0$, we have

$$
\sqrt{2}\left(x+\frac{1}{2}\right)^{x+1 / 2} e^{-x} \leq \Gamma(x+1) \leq e^{\gamma / e^{\gamma}}\left(x+\frac{1}{e^{\gamma}}\right)^{x+1 / e^{\gamma}} e^{-x},
$$




$$
\sqrt{2 e}\left(\frac{x+1 / 2}{e}\right)^{x+1 / 2} \leq \Gamma(x+1)<\sqrt{2 \pi}\left(\frac{x+1 / 2}{e}\right)^{x+1 / 2}
$$

and

$$
\begin{aligned}
& \sqrt{2 x+1} x^{x} \exp \left\{-\left[x+\frac{1}{6(x+3 / 8)}-\frac{4}{9}\right]\right\}<\Gamma(x+1) \\
&<\sqrt{\pi(2 x+1)} x^{x} \exp \left\{-\left[x+\frac{1}{6(x+3 / 8)}\right]\right\} .
\end{aligned}
$$

By the software Mathematica Version 7.0.0, we can reveal that

(1) the double inequalities (1.6) and (4.7) do not include each other on $(0,1)$,

(2) the right-hand side inequality in (1.6) is better than the one in (4.8) on $(0,1)$,

(3) the left-hand side inequalities in (1.6) and (4.8) are not included each other on $(0,1)$,

(4) the lower bound in (1.6) improves the corresponding one in (4.9), but the right-hand side inequalities in (1.6) and (1.6) do not contain each other on $(0,1)$.

Remark 4. It is clear that when $x \in \mathbb{N}$ the inequality (1.7) becomes equality. This shows us that for $x>1$ the double inequality (1.7) is better than those double inequalities listed in the above Remarks 2 and 3.

Remark 5. In [15, Theorem 1], among other things, it was proved that the function

$$
F(x)=\frac{\ln \Gamma(x+1)}{x \ln (2 x)}
$$

is both strictly increasing and strictly concave on $\left(\frac{1}{2}, \infty\right)$. By L'Hospital Rule and the double inequality $(2.1)$ for $k=1$, we obtain

$$
\lim _{x \rightarrow \infty} F(x)=\lim _{x \rightarrow \infty} \frac{\psi(x+1)}{1+\ln (2 x)}=\lim _{x \rightarrow \infty}\left[x \psi^{\prime}(x+1)\right]=1,
$$

so it follows that $\Gamma(x+1)<(2 x)^{x}$ on $\left(\frac{1}{2}, \infty\right)$, which is not better than the right-hand side inequality in $(1.6)$ on $\left(\frac{1}{2}, 1\right)$.

Remark 6. By similar argument to the proof of Theorem 1, we may prove that the function

$$
\frac{\ln \Gamma(x+1)}{\ln \left(x^{2}+6\right)-\ln (x+6)}
$$

is strictly decreasing on $(0,1)$. Consequently,

$$
\left(\frac{x^{2}+6}{x+6}\right)^{6 \gamma}<\Gamma(x+1)<\left(\frac{x^{2}+6}{x+6}\right)^{7(1-\gamma)}, \quad x \in(0,1) .
$$

Motivating by monotonic properties of the functions (1.3) and (4.11), we pose the following open problem: What is the largest number $\lambda>1$ (or the smallest number $\lambda<6$ respectively) for the function

$$
\frac{\ln \Gamma(x+1)}{\ln \left(x^{2}+\lambda\right)-\ln (x+\lambda)}
$$

to be strictly increasing (or decreasing respectively) on $(0,1)$ ?

Remark 7. Finally, we pose the following conjectures. 
(1) The function (1.3) is strictly increasing not only on $(0,1)$ but also on $(0, \infty)$.

(2) For $\tau>0$, the function

$$
\begin{cases}\frac{\ln \Gamma(x)}{\ln \left(x^{2}+\tau\right)-\ln (x+\tau)}, & x \neq 1 \\ -(1+\tau) \gamma, & x=1\end{cases}
$$

is strictly increasing with respect to $x \in(0, \infty)$.

(3) Recall from [11, Chapter XIII], [20, Chapter 1] or [21, Chapter IV] that a function $f$ is completely monotonic on an interval $I$ if $f$ has derivatives of all orders on $I$ and

$$
0 \leq(-1)^{n} f^{(n)}(x)<\infty
$$

for $x \in I$ and $n \geq 0$. We conjecture that the function

$$
h(x)= \begin{cases}\frac{\ln x}{\ln \left(1+x^{2}\right)-\ln (1+x)}, & x \neq 1 \\ 2, & x=1\end{cases}
$$

is completely monotonic on $(0, \infty)$.

Remark 8. For the history, backgrounds, origins, developments of bounding the gamma function, please refer to the expository and survey article [12] and plenty of references therein.

Remark 9. This paper is a revised version of the preprint [14].

Acknowledgements. The authors appreciate anonymous referees for their helpful and valuable comments on this paper.

\section{REFERENCES}

[1] H. Alzer, Inequalities for the gamma function, Proc. Amer. Math. Soc. 128 (1999), no. 1, $141-147.5$

[2] H. Alzer and N. Batir, Monotonicity properties of the gamma function, Appl. Math. Lett. 20 (2007), no. 7, 778-781; Available online at http://dx.doi.org/10.1016/j.aml.2006.08.026. 5

[3] G. D. Anderson, M. K. Vamanamurthy, and M. Vuorinen, Special functions of quasiconformal theory, Expo. Math. 7 (1989), 97-136. 2

[4] N. Batir, Inequalities for the gamma function, Arch. Math. 91 (2008), 554-563. 5

[5] B.-N. Guo, R.-J. Chen, and F. Qi, A class of completely monotonic functions involving the polygamma functions, J. Math. Anal. Approx. Theory 1 (2006), no. 2, 124-134. 3

[6] B.-N. Guo and F. Qi, Some properties of the psi and polygamma functions, Hacet. J. Math. Stat. 39 (2010), 219-231. 3

[7] B.-N. Guo and F. Qi, Two new proofs of the complete monotonicity of a function involving the psi function, Bull. Korean Math. Soc. 47 (2010), no. 1, 103-111; Available online at http://dx.doi.org/10.4134/BKMS.2010.47.1.103. 3

[8] B.-N. Guo, F. Qi and H. M. Srivastava, Some uniqueness results for the non-trivially complete monotonicity of a class of functions involving the polygamma and related functions, Integral Transforms Spec. Funct. 21 (2010), no. 11, 103-111; Available online at http://dx.doi.org/ 10.1080/10652461003748112. 3

[9] B.-N. Guo, Y.-J. Zhang and F. Qi, Refinements and sharpenings of some double inequalities for bounding the gamma function, J. Inequal. Pure Appl. Math. 9 (2008), no. 1, Art. 17; Available online at http://www.emis.de/journals/JIPAM/article953.html?sid=953. 5

[10] P. Ivády, A note on a gamma function inequality, J. Math. Inequal. 3 (2009), no. 2, 227-236. 1

[11] D. S. Mitrinović, J. E. Pečarić, A. M. Fink, Classical and New Inequalities in Analysis, Kluwer, Dordrecht, 1993. 7 
[12] F. Qi, Bounds for the ratio of two gamma functions, J. Inequal. Appl. 2010 (2010), Article ID 493058, 84 pages; Available online at http://dx.doi.org/10.1155/2010/493058. 3, 7

[13] F. Qi and B.-N. Guo, A logarithmically completely monotonic function involving the gamma function, Taiwanese J. Math. 14 (2010), no. 4, 1623-1628. 3

[14] F. Qi and B.-N. Guo, An elegant refinement of a double inequality for the gamma function, Available online at http://arxiv.org/abs/1001.1495. 7

[15] F. Qi and B.-N. Guo, Monotonicity and logarithmic convexity relating to the volume of the unit ball, Available online at http://arxiv.org/abs/0902.2509. 6

[16] F. Qi and B.-N. Guo, Necessary and sufficient conditions for functions involving the triand tetra-gamma functions to be completely monotonic, Adv. Appl. Math. 44 (2010), no. 1, 71-83; Availale online at http://dx.doi.org/10.1016/j.aam.2009.03.003. 3

[17] F. Qi, S. Guo and B.-N. Guo, Complete monotonicity of some functions involving polygamma functions, J. Comput. Appl. Math. 233 (2010), no. 9, 2149-2160; Available online at http: //dx.doi.org/10.1016/j.cam.2009.09.044. 3

[18] F. Qi, D.-W. Niu and B.-N. Guo, Refinements, generalizations, and applications of Jordan's inequality and related problems, J. Inequal. Appl. 2009 (2009), Article ID 271923, 52 pages; Available online at http://dx.doi.org/10.1155/2009/271923. 2

[19] F. Qi and A. Sofo, An alternative and united proof of a double inequality for bounding the arithmetic-geometric mean, Politehn. Univ. Bucharest Sci. Bull. Ser. A Appl. Math. Phys. 71 (2009), no. 3, 69-76. 2

[20] R. L. Schilling, R. Song and Z. Vondraček, Bernstein Functions, de Gruyter Studies in Mathematics 37, De Gruyter, Berlin, Germany, 2010. 7

[21] D. V. Widder, The Laplace Transform, Princeton University Press, Princeton NJ, 1946. 7

(F. Qi) Department of Mathematics, College of Science, Tianjin Polytechnic UniverSity, Tianjin City, 300160, China

E-mail address: qifeng618@gmail.com, qifeng618@hotmail.com, qifeng618@qq.com

$U R L:$ http://qifeng618. wordpress.com

(B.-N. Guo) School of Mathematics and Informatics, Henan Polytechnic University, Jiaozuo City, Henan Province, 454010, China

E-mail address: bai.ni.guo@gmail.com, bai.ni.guo@hotmail.com 